\title{
Model based optimization criteria for the generation of deep compressive residual stress fields in high elastic limit metallic alloys by ns-laser shock processing
}

\author{
M. Morales , J.L. Ocaña, C. Molpeceres, J.A. Porro, A. García-Beltrán \\ Centro Láser UPM, Universidad Politécnica de Madrid, Spain \\ Campus Sur UPM, Edificio La Arboleda Ctra. de Valencia, km. 7,300, 28031 Madrid, Spain
}

\begin{abstract}
Laser Shock Processing (LSP) is based on the application of a high intensity pulsed Laser beam (I>1 GW/ $/ \mathrm{cm}^{2} ; \tau<50 \mathrm{~ns}$ ) on a metallic target forcing a sudden vaporization of its surface into a high temperature and density plasma that immediately develops inducing a shock wave propagating into the material.

The main acknowledged advantages of LSP consist on its capability of inducing a relatively deep compression residual stresses field into metallic alloy pieces allowing an improved mechanical behavior, explicitly, the life improvement of the treated specimens against wear, crack growth and stress corrosion cracking. Due to these specific advantages, Laser Shock Processing is considered as a competitive alternative technology to classical treatments for improving fatigue, corrosion cracking and wear resistance of metallic materials, and is being developed as a practical process amenable to production technology.

In this paper, a model based systematization of process optimization criteria and a practical assessment on the real possibilities of the technique is presented along with practical results at laboratory scale on the application of LSP to characteristic high elastic limit metallic alloys, showing the induced residual stresses fields and the corresponding results on mechanical properties improvement induced by the treatment. The homogeneity of the residual stress fields distribution following the laser treatment spatial density will be specially analyzed.
\end{abstract}

Keywords: Plasma; Laser Shock Processing; Surface treatment; Shock waves; Residual stress; Numerical modeling

\section{Introduction}

Following its first developments in the 1970s laser shock processing (LSP) is being consolidating as an effective technology for the improvement of surface mechanical and corrosion resistance properties of metals and is being developed as a practical process amenable to production engineering

However, although significant work from the experimental side has contributed to explore the optimum conditions of application of the treatments only limited attempts have been developed in the way of full comprehension and predictive assessment of the characteristic physical processes
Additionally, some relevant work has been made in the line of prediction and characterization of the mechanical properties enhancement of material treated by the LSP technique [8-10].

A fundamental reason for the referred lack of predictive capability of LSP processes is their inherent physical complexity, specially stemming on the coexistence of different material phases (including plasma) developing and interacting under the action of the high intensity laser beam.

In the present paper, a review on the physical issues dominating the development of LSP processes from a high intensity laser-matter interaction point of view is presented along with the theoretical and computational methods developed by the authors for their predictive assessment, and practical results at laboratory scale on the application of the technique are shown along with corresponding results on the mechanical properties improvement. 


\section{Physical basis and theoretical modelling of LSP processes}

\subsection{Background: physical basis of LSP processes}

Laser Shock Processing (LSP) is based on the application of a high intensity pulsed Laser beam (I> $\left.1 \mathrm{GW} / \mathrm{cm}^{2} ; \tau<50 \mathrm{~ns}\right)$ on a metallic target forcing a sudden vaporization of its surface into a high temperature and density plasma that immediately develops inducing a shock wave propagating into the material.

During a first step (during which the laser beam is active on the piece), the laser energy is deposited at the interface between the target and the surrounding medium (normally a transparent confining material). The pressure generated by the plasma induces two shock waves propagating in opposite directions (inside the target and towards the confining material respectively). When the laser is switched off, the plasma continues to maintain a pressure which decreases during its expansion as a consequence of the increase of the plasma volume. Finally, for longer times, after the plasma recombination, the projectile-like expansion of the heated gas inside the interface adds further mechanical momentum to the target.

The pressure induced by the laser generated shock wave into the treated material, which is the direct responsible for the achieved material permanent deformation, has to be optimized as a function of the laser characteristics and other process parameters.

The description of the relevant laser absorption phenomena becomes hardly complicated because of the non-linear effects appearing along the interaction process and which significantly alter the shocking dynamics. These include ionization, the resulting electronic population dynamics leading to modified absorption properties, thermal fluxes and fluid dynamic motion and, eventually, generation of electromagnetic radiation fields and energy transport by suprathermal particles.
The effective presence of a deleterious effect induced by the thermal flux generated upon laser material interaction and, on the other, the very limited extent of this influence to a narrow layer under the target free surface, what, however, is of a critical importance from the point of view of the mechanical behaviour of the treated piece and deserves a further detailed evaluation aiming process optimization

Additionally, from the practical point of view, the effect of parasitic breakdown plasma occurring in the confining medium is highly deleterious for the process so that it has to be explicitly prevented.

\subsection{The SHOCKLAS Model for the analysis and predictive assessment of LSP processes}

The appropriate description of the process requires a threelevel description providing the adequate interconnection of the data obtained in each phase in a self-consistent way from the physical point of view.

The referred three-level description includes:

i) Analysis of the plasma electronic population dynamics, including consideration of breakdown phenomenology in dielectric media,

ii) Simulation of the hydrodynamic phenomenology arising from plasma expansion between the confinement layer and the base material

iii) Analysis of the propagation and induction of permanent structural changes by shock wave evolution in bulk material

A calculational model dealing with these three main aspects of the process modelling in a coupled way has been developed

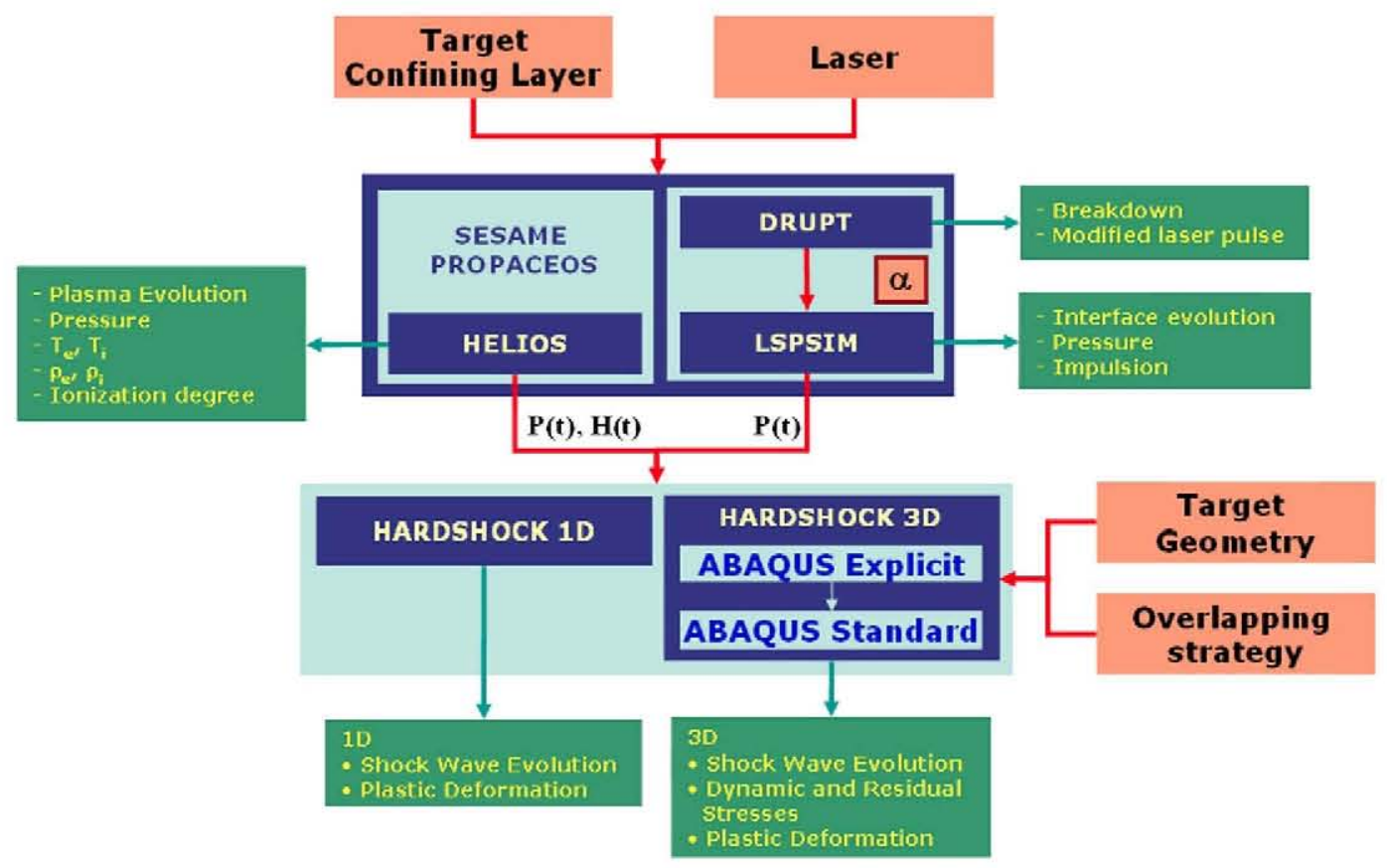

Fig. 1. Calculation scheme of the developed coupled model for the analysis of Laser Shock Processing experiments. 
by the authors. The developed calculational model (SHOCKLAS) is integrated by three principal modules, respectively called HELIOS, LSPSIM and HARDSHOCK, and has been conceived for the analysis of the problem of laser shock waves generation and propagation under three different but intercomplementary approaches In Fig. 1, a schematic representation is shown on the way of coupling of the referred modules.

HELIOS is a 1-D radiation-magnetohydrodynamics code that is used to simulate the dynamic evolution of laser created plasmas HELIOS solves Lagrangian hydrodynamics equations for a single fluid. Electrons and ions are assumed to be co-moving. Pressure contributions to the equation of motion come from electrons, ions, radiation, and the magnetic field. Energy transport in the plasma can be treated using either a onetemperature $\left(T_{\mathrm{i}}=T_{\mathrm{e}}\right)$ or two-temperature $\left(T_{\mathrm{i}} \neq T_{\mathrm{e}}\right)$ model. Both the electrons and ions are assumed to have Maxwellian distributions defined by their respective temperatures. Material EOS and opacities properties are based on either SESAME tables or PROPACEOS tables.

Laser energy deposition is computed using an inverse Bremsstrahlung model, with the restriction that no energy in the beam passes beyond the critical surface.

LSPSIM is a one-dimensional model intended for the estimation of the pressure wave applied to the target material in Laser Shock experiments [7,10]. LSPSIM obtains the targetconfining medium gap amplitude, by solving the coupled system of energy and impulse equations subject to the thermofluid dynamic conditions imposed by the laser energy deposition. In this model the fraction of laser energy invested in ionization processes $(1-\alpha)$ is a experimental value.

On the basis of the time-dependent pressure profile calculated by HELIOS or LSPSIM, HARDSHOCK solves the shock propagation problem into the solid material, with specific consideration of the material response to thermal and mechanical alterations induced by the propagating wave itself (i.e. effects as elastic-plastic behaviour, changes in elastic constants, phase changes, etc.).

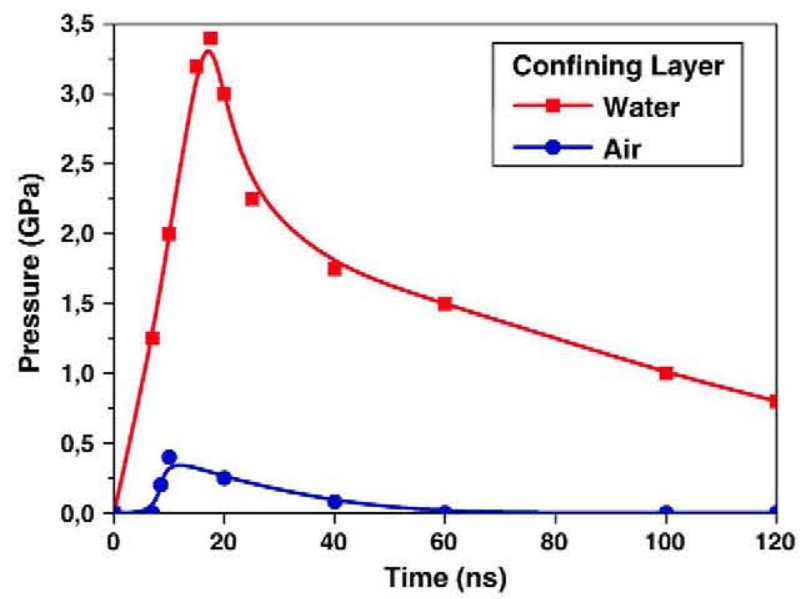

Fig. 2. Plasma pressure obtained with HELIOS for a Aluminium/Water plasma and Aluminium/Air plasma in LSP conditions.

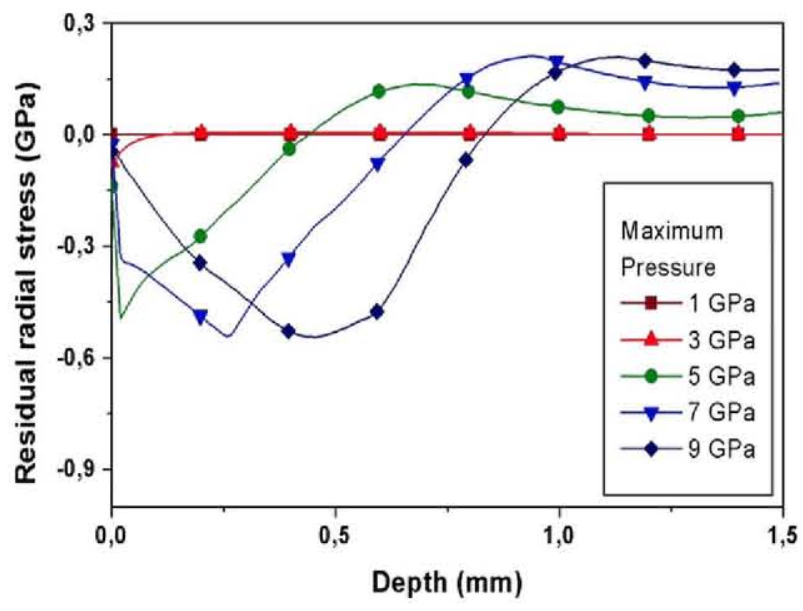

Fig. 3. Depth distribution of radial stress in Ti6Al4 V subject to the application of $1 \mathrm{~mm}$ radius pressure pulses of $25 \mathrm{~ns}$ FWHM and different peak pressures.

The target material subject to LSP is heated due to two main mechanisms: direct laser interaction heating and heating by plastic deformation work. The resulting temperature rise is correspondingly computed, so that this temperature change produces, in turn, a local thermal expansion of the target material whose subsequent thermal strains have to be consistently calculated.

For this kind of problems, a 3D version, based in the FEM commercial code ABAQUS ${ }^{\circledR}$ is used. From the point of view of time differencing, the usual strategy of explicit differencing for the initial fast shock propagation phase followed by standard implicit differencing for the analysis of the final residual stresses equilibrium is used.

The 3D simulated geometry can be change in order to properly adjust to experimental conditions. The FEM element used for the mechanical simulation is C3D8R a 8-node brick reduced integration with hourglass control.

\section{Model results}

The described model has been applied to the simulation of the material behaviour of relevant metal alloys subject to LSP conditions. The final effect on the solid material of gaussian laser pulses incident with given maximum intensity and duration is analyzed. With the aid of HELIOS and LSPSIM, the resulting plasma pressure applied to the (coated and supposed undamaged) solid material is obtained for representative breakdownfree conditions (see Fig. 2), the influence of the characteristic laser parameters and process development being characterized.

In Fig. 2 plasma pressure obtained with HELIOS for a Aluminium/Water plasma and Aluminium/Air plasma in LSP conditions are displayed. The effect of water confinement can be clearly observed.

An important fact in these results (thanks to the realistic character of the performed axissymmetric 2D calculations) is the potentially deleterious effect of the transient effects induced by a continuously exerted pressure on material already shocked. This is a clear result showing the effective coupled spatialtemporal dependence of LSP processes and their resulting difficulty for optimization in practical applications. 


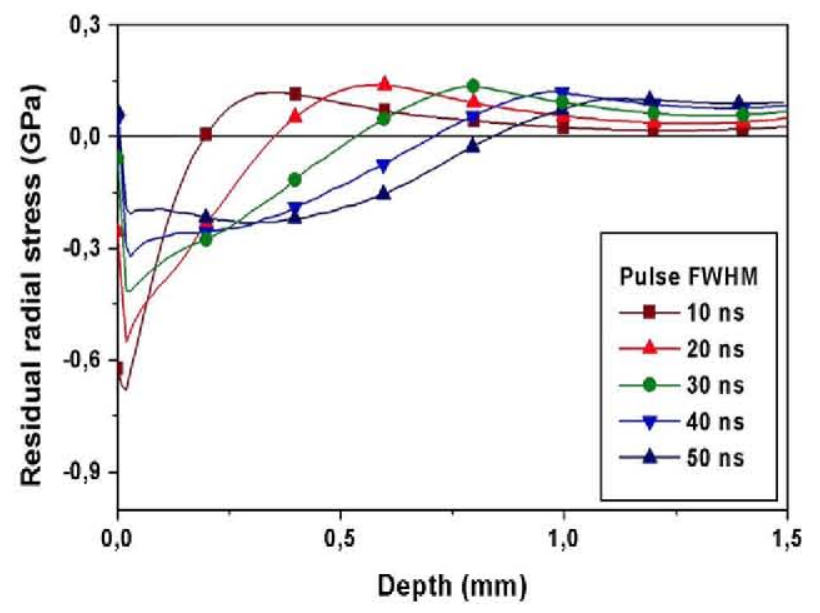

Fig. 4. Depth distribution of radial stress in Ti6Al4 V subject to the application of $1 \mathrm{~mm}$ radius pressure pulses of $5 \mathrm{GPa}$ peak pressure and different FWHM's.

3.1. Analysis of the influence of pressure pulse FWHM and peak intensity on the maximum stress level and penetration of $2 D$ axissymmetric shocks

In Fig. 3 the effect of the modification of the maximum pulse pressure on the depth distribution of radial residual stress and the maximum compressive residual stress reached are analyzed.

In Fig. 4 so does the effect of the modification of the pressure pulse FWHM on the depth distribution of radial residual stress and the maximum compressive residual stress.

3.2. Analysis of the influence of the shocked surface size on the maximum stress level and penetration of axissymmetric pressure pulses

A second effect analyzed through the application of the described FEM model in axissymmetric 2D geometry has been that of energy delocalization due to a finite laser spot size. In

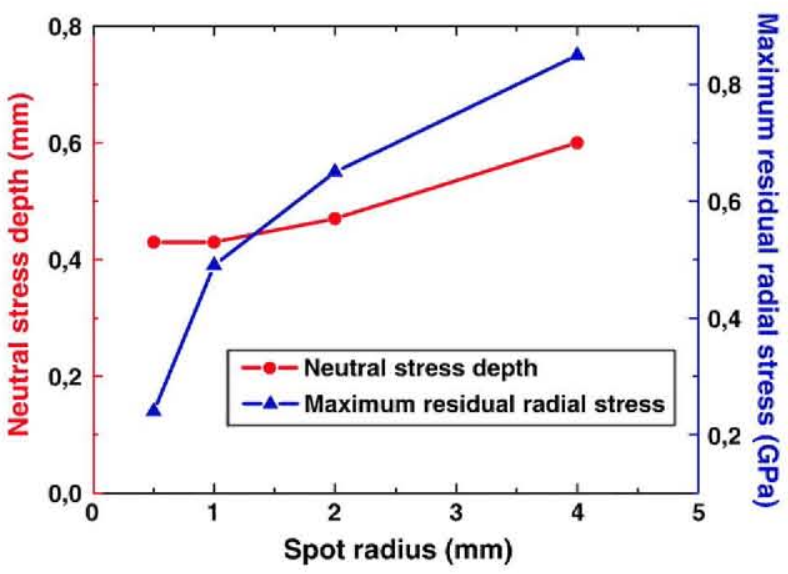

Fig. 6. Neutral stress depth and maximum compressive residual stress reached in Ti6Al4 V subject to pressure pulses of 5 GPa peak pressure and $25 \mathrm{~ns}$ FWHM for different values of shocked area radii.

Figs. 5-6, calculational results on the evolution and final results of radial stress induced in Ti6Al4 $\mathrm{V}$ by pressure pulses applied over different surface sizes are shown.

In this case, as a consequence of the combination of two main coupled mechanisms, namely the regular shock wave attenuation due to energy released for plastic deformation and its attenuation due to the arrival of lateral rarefaction waves coming from the shocked zone outer sides, characteristic effects can be observed in the maximum radial residual stress attained and in the neutral stress penetration depth. While residual stresses clearly increase with the shocked surface radius (an effect typically expected because of the higher values of total energy involved), the increase of the neutral stress penetration depth is generally inhibited because of the deleterious effect of the second attenuation mechanism. This, again, reveals the dynamic and multidimensional character of the LSP treatment and its need for analysis with realistic calculational tools of the level described here.

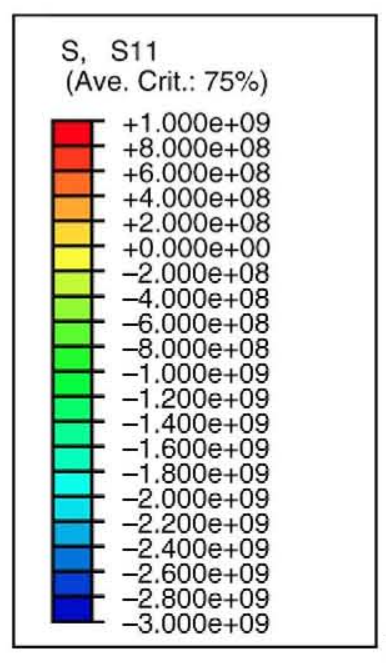

Spot radius $(\mathrm{mm})=$

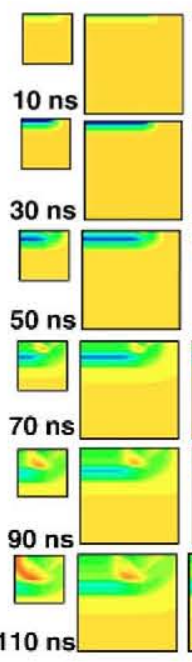

0,5

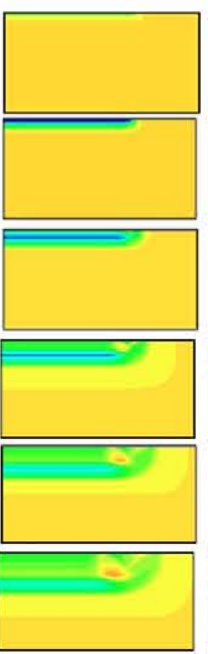

2

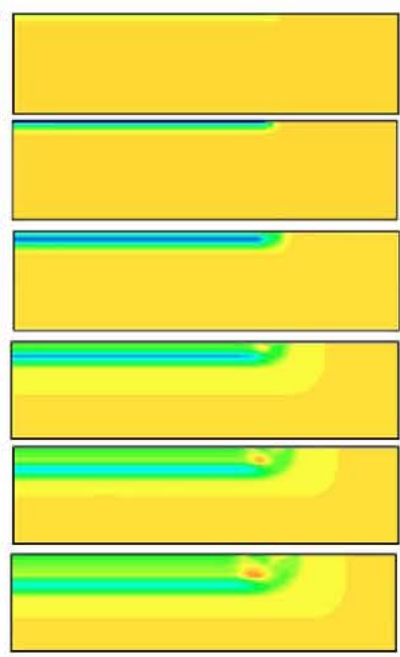

4

Fig. 5. Time evolution of radial stress induced in Ti6Al4 V by pressure pulses applied over different surface sizes. 
3.3. Evaluation of the residual stress and deformation obtained by the application of adjacent pulses covering an extended surface

Overlapping technique produces the effect of the progressive saturation of shocked zones as far as the effect of multiple pressure pulses reach them. At the same time, and as a consequence, the progressive homogeneization of the residual stresses field is obtained in the zones for which an important number of impacts have been applied, what is especially important from the experimental point of view. A sample of the analysis possibilities of the described model in full 3D geometries is shown in Fig. 8.

\section{Experimental setup of LSP applications}

The practical irradiation system used as experimental in LSP treatments conducted at CLUPM is schematically and photographically shown in Fig. 7. The LSP experiments reported in this paper were performed on A12024-T351 alloy at $1064 \mathrm{~nm}$ laser wavelength using a $\mathrm{Q}$ switched $\mathrm{Nd}$ :YAG laser operating at $10 \mathrm{~Hz}$ and providing $9.4 \mathrm{~ns}$ FWHM, 1.2 J pulses. A convergent lens was used to deliver the laser energy over a $1.5 \mathrm{~mm}$ spot diameter. The confining layer was provided by a water jet incident close to the laser interaction zone. The test piece is fixed on a holder and is driven along $X$ and $Y$ directions by means of a computer controlled stage needed for the irradiation of extended areas of material following a pre-defined pulse overlapping strategy [4].

\section{Experimental results}

Residual stress distribution was determined according to the ASTM E837-01 Standard test method for determining residual stresses by the hole drilling strain gage method. A12024-T351 specimens $8 \mathrm{~mm}$ thick were used for the experiments. Fig. 8
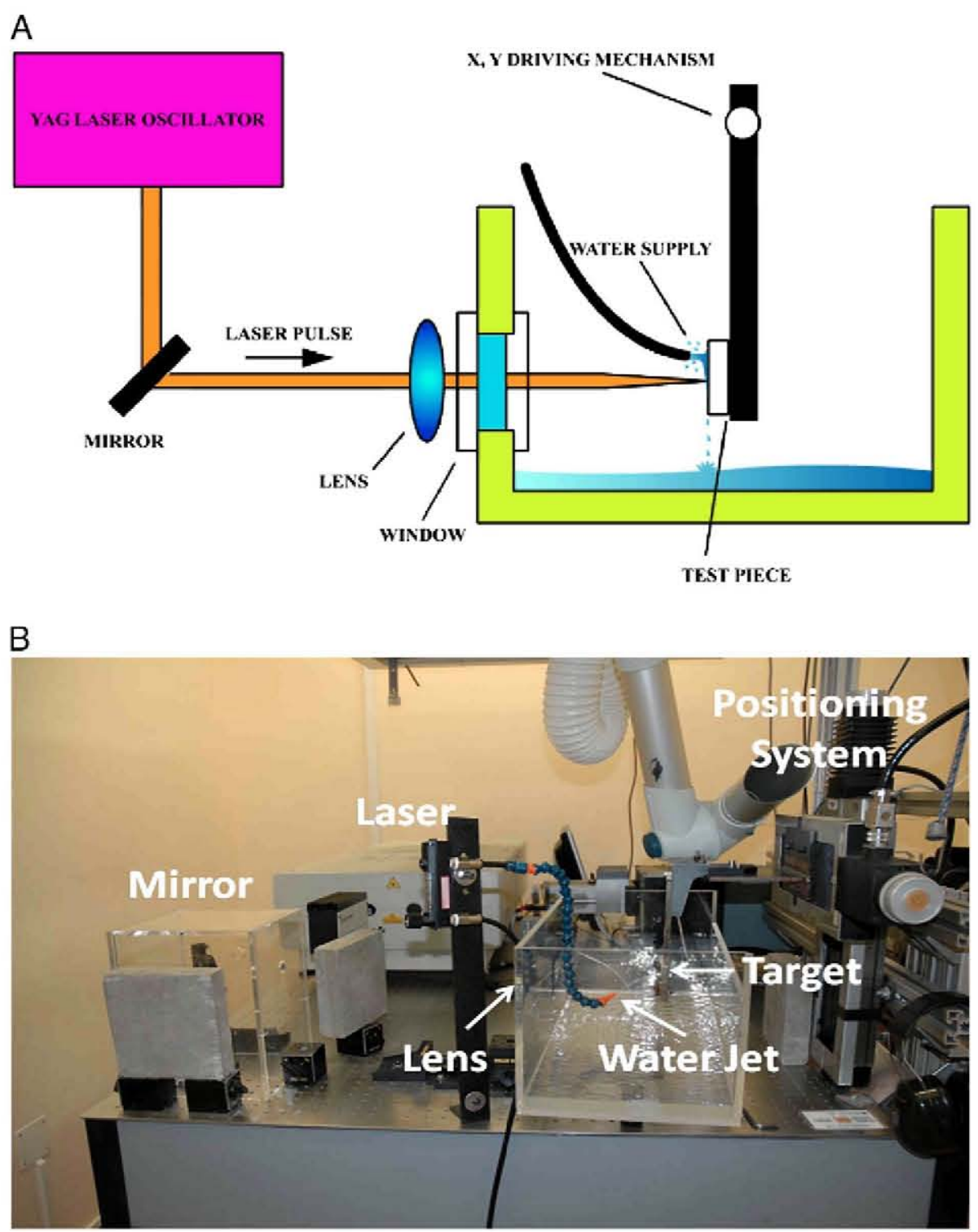

Fig. 7. Schematic representation and photographic view of the LSP irradiation experimental setup. 


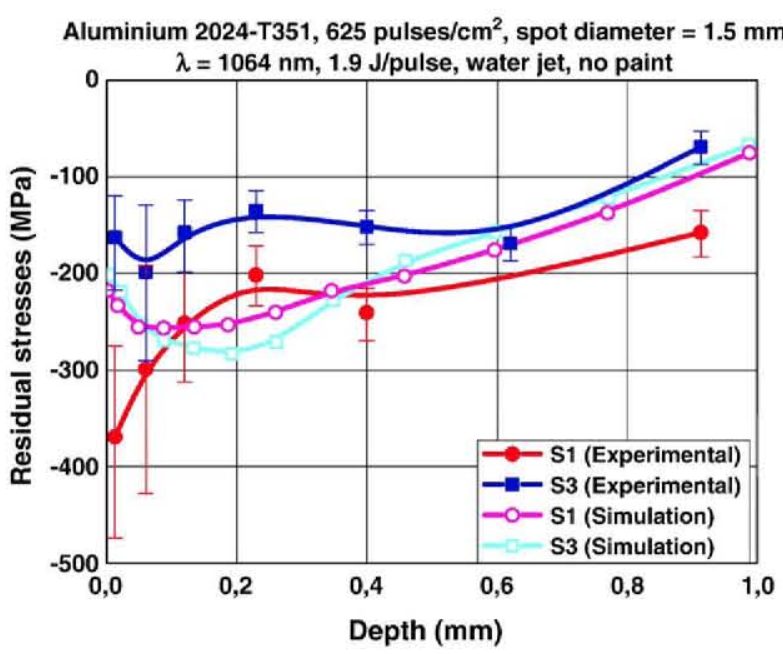

Fig. 8. Comparison of residual stresses along $z$-coordinate induced in $\mathrm{Al} 2024$ T351 by the LSP technique with simulation results.

shows, as an example, the depth profiles obtained for LSP-induced residual stresses and a comparison with simulation results.

\section{Discussion}

The phenomenology involved in LSP processes is complicated mostly because of their characteristic laser-plasma interaction dynamics. The need for a practical capability of process control in practical applications has led to the development of comprehensive theoretical/computational models for the predictive assessment of the complex phenomenology involved.

From the practical point of view, the LSP technology allows the effective induction of residual stresses fields in metallic materials. Experimental results obtained with commercial Q-switched lasers prove complete feasibility at laboratory scale. There still remains the need for commercially available laser systems to turn LSP productive enough in order to become a technology competitive to present day techniques used for similar purposes, namely shot peening, for the treatment of heavy duty components in the aeronautical, nuclear and automotive industries.

\section{Acknowledgements}

Work partly supported by MCYT (Spain; Project DPI200509152-C02-01) and EADS-Spain.

\section{References}

B.P. Fairand, B.A. Wilcox, W.J. Gallagher, D.N. Williams, J. Appl. Physi. 43 (1972) 3893

Y. Sano, N. Mukai, K. Okazaki, M. Obata, Nucl. Instrum. Methods Phys. Res., B Beam Interact. Mater. Atoms 121 (1997) 432.

P. Peyre, P. Merrien, H.P. Lieurade, R. Fabbro, Surf. Eng. 11 (1995) 47.

J.L. Ocaña, C. Molpeceres, J.A. Porro, G. Gómez, M. Morales, Appl. Surf. Sci. 238 (2004) 501.

R.D. Griffin, B.L. Justus, A.J. Campillo, L.S. Goldberg, J. Appl. Physi. 59 (1986) 1968.

L. Berthe, R. Fabbro, P. Peyre, L. Tollier, E. Bartnicki, J. Appl. Physi. 82 (1997) 2826.

J.L. Ocaña, C. Molpeceres, M. Morales, A. García-Beltrán, in: C.R. Phipps, M. Niino (Eds.), High-Power Laser Ablation II SPIE Proceedings, 3885,2000 , p. 252.

W. Braisted, R. Brockman, Int. J. Fatigue 21 (1999) 719.

W. Zhang, Y.L. Yao, J. Manuf. Sci. Eng. 124 (2002) 369.

J.L. Ocaña, M. Morales, C. Molpeceres, J. Torres, Appl. Surf. Sci. 238 (2004) 242

J.L. Ocaña, M. Morales, C. Molpeceres, J.A. Porro, A. García-Beltrán, Mat. Sci. Forum 539-543 (2007) 1116.

D. Devaux, R. Fabbro, L. Tollier, E. Bartnicki, J. Appl. Physi. 74 (1993) 2268 .

J.J. MacFarlane, I.E. Golovkin, P.R. Woodruff, J. Quant. Spectrosc. Radiat. Transfer 99 (2006) 381.

S.P. Lyon, J.D. Johnson, "SESAME: The Los Alamos National Laboratory Equation of State Database", Technical report, LA-UR-92-3407, Los Alamos National Laboratory, Los Alamos, NM, 1992. 